\title{
Generation of live rat offspring by intrauterine insemination with epididymal spermatozoa cryopreserved at $-196^{\circ} \mathrm{C}$
}

\author{
E. Nakatsukasa ${ }^{1}$, T. Inomata ${ }^{2}$, T. Ikeda ${ }^{3}$, M. Shino ${ }^{1}$ and \\ N. Kashiwazaki ${ }^{1 *}$ \\ ${ }^{1}$ Laboratory of Animal Reproduction, School of Veterinary Medicine, Azabu University, \\ Sagamihara, 229-8501 Japan; ${ }^{2}$ Laboratory of Laboratory Animals, School of Veterinary \\ Medicine, Azabu University, Sagamihara, 229-8501 Japan; and ${ }^{3}$ Laboratory of Microbiology, \\ School of Veterinary Medicine, Azabu University, Sagamihara, 229-8501 Japan
}

This study reports the development of a reliable method for cryopreservation of rat epididymal spermatozoa and the production of live young by artificial insemination using these cryopreserved spermatozoa. The motility and membrane integrity of rat spermatozoa were investigated after spermatozoa had been subjected to physical stress and frozen with various concentrations of glycerol $(0,3$ and $6 \%$ ) either in the presence or absence of Equex Stem as cryoprotective agents. The ability of cryopreserved spermatozoa to generate normal offspring by intrauterine insemination was also evaluated. Rat spermatozoa that had been centrifuged at $700 \mathrm{~g}$ for $5 \mathrm{~min}$ showed a significant decrease in motility compared with non-centrifuged spermatozoa. In addition, after centrifugation three times the percentage of membrane-intact spermatozoa decreased to approximately $0 \%$. The percentage of membrane-intact spermatozoa was significantly higher $(P<0.01)$ in semen samples that had been frozen in medium without glycerol than in samples frozen in medium with $3 \%$ glycerol. Although the addition of $0.7 \%$ Equex Stem to medium without glycerol or with $3 \%$ glycerol did not influence rates of sperm motility after freezing and thawing, the percentage of membrane-intact spermatozoa was improved by the presence of $0.7 \%$ Equex $(P<0.05)$. Therefore, rat spermatozoa were handled gently to avoid physical stress and were frozen in medium containing $23 \%$ egg yolk, $8 \%$ lactose monohydrate and $0.7 \%$ Equex Stem, at pH 7.4 adjusted with $10 \%$ Tris(hydroxymethyl)aminomethane solution. Thirteen female rats were inseminated into the oviductal end of both uterine horns with frozen-thawed spermatozoa. Forty-one normal live offspring were obtained from nine of the inseminated females. These results indicate that frozen-thawed rat spermatozoa can generate normal offspring. To our knowledge, this procedure is the first successful production of offspring using spermatozoa cryopreserved in liquid nitrogen.

\section{Introduction}

With the exception of the mouse, the rat is considered to be one of the most important and valuable laboratory animals for research in biology, physiology and medicine. Therefore, the need for methods to cryopreserve rat spermatozoa and to preserve rat mutants, including transgenic strains, in the form of cryopreserved spermatozoa is widely recognized. In mice, a large number of various mutant and transgenic strains have been established in different laboratories. Cryopreservation of spermatozoa is an effective, simple and cost effective method to maintain these strains (Nakagata, 1996). Methods for cryopreserving mouse spermatozoa have already been developed (Nakagata et al., 1997; An et al., 2000; Sztein et al., 2000); however, to date, successful cryopreservation of rat spermatozoa has not been studied.

*Correspondence

Email: nkashi@azabu-u.ac.jp
The objective of the present study was to develop a reliable and reproducible method for cryopreserving rat spermatozoa in liquid nitrogen. The motility and membrane integrity of rat epididymal spermatozoa subjected to physical stress and frozen with various concentrations of glycerol with or without Equex Stem as a cryoprotective agent were investigated. The ability of cryopreserved spermatozoa to generate normal offspring by intrauterine insemination was also evaluated.

\section{Materials and Methods}

Animals and semen collection

Rats used in the present study were maintained in accordance with the guidelines of the Laboratory Animal Committee of Azabu University.

Sixty-four mature SD (Jcl: SD) male rats (15-30 weeks old) were used as semen donors; 16 vasectomized $\mathrm{BN}$ (BN/Sea) male rats (15 weeks old) were used to induce pseudopregnancies; and 59 mature Wistar (Jla: Wistar) 
female rats (8-18 weeks old) acted as recipients of inseminations. Mature male rats were killed by cervical dislocation and their epididymides were excised. Epididymides collected from each donor were placed in a plastic dish containing $3 \mathrm{ml}$ medium I. Medium I, which was used for freezing rat spermatozoa, was modified from NSF-I media used by Kikuchi et al. (1999) to freeze pig spermatozoa. Medium I contained 23\% (v/v) egg yolk, $8 \%$ $(\mathrm{w} / \mathrm{v})$ lactose monohydrate and antibiotics (1000 iu penicillin $\mathrm{G}$ potassium $\mathrm{ml}^{-1}, 1 \mathrm{mg}$ streptomycin sulphate $\mathrm{ml}^{-1}$; Sigma, St Louis) at $\mathrm{pH} 7.4$, adjusted with $10 \%(\mathrm{w} / \mathrm{v})$ Tris(hydroxymethyl)aminomethane (Sigma) solution. The epididymides were dissected with eye scissors to obtain epididymal spermatozoa. Tissue debris was removed from the droplet of medium. Sperm motility was evaluated by placing a small drop of medium into an examination chamber, placed on a micro-warm-plate at $37^{\circ} \mathrm{C}$, and then examined under a light microscope at $\times 100$. The number of spermatozoa in the samples was determined in duplicate by direct examination under the microscope using a haemocytometer (a Thoma chamber). Only semen with a sperm motility $>50 \%$ and sperm concentrations of $1-3 \times 10^{8}$ cells $\mathrm{ml}^{-1}$ was used in the present study.

\section{Centrifugation of semen}

Epididymal spermatozoa were collected in $3 \mathrm{ml}$ of rat 1 cell embryo culture medium (R1ECM; Miyoshi et al., 1997) using the method described above to examine the effect of centrifugation on the motility and membrane integrity of rat spermatozoa. Sperm suspensions were dispensed in $1 \mathrm{ml}$ aliquots into $15 \mathrm{ml}$ centrifuge tubes and then centrifuged at $700 \mathrm{~g}$ for $5 \mathrm{~min}$. Between each centrifugation treatment, sperm pellets were resuspended by pipetting with a micropipette (Pipetman P-1000, Gilson Inc., Middleton, WI) attached to a blue tip (Gilson Inc.). The motility and membrane integrity of spermatozoa centrifuged once, twice or three times were assessed.

\section{Freezing and thawing semen}

Semen samples in $3 \mathrm{ml}$ medium I were maintained at $15^{\circ} \mathrm{C}$ for $30 \mathrm{~min}$ and then at $5^{\circ} \mathrm{C}$ for $30 \mathrm{~min}$. The cooled sperm suspension in medium I was added to $3 \mathrm{ml}$ medium II that had been cooled at $5^{\circ} \mathrm{C}$. Medium II consisted of medium I either with or without 1.4\% (v/v) Equex Stem (Nova Chemical Sales, Inc., Scituate, MA) and with either 0, 6 or $12 \%(\mathrm{v} / \mathrm{v})$ glycerol (Merk, Darmstadt). Final glycerol concentrations in the freezing media were 0,3 or $6 \%(\mathrm{v} / \mathrm{v})$, respectively. Semen cooled at $5^{\circ} \mathrm{C}$ was loaded into $0.25 \mathrm{ml}$ plastic straws (Fujihira Inc.) and exposed to liquid nitrogen vapour at $-170^{\circ} \mathrm{C}, 2 \mathrm{~cm}$ above the liquid nitrogen level for $10 \mathrm{~min}$. The straws were maintained on $0.2 \mathrm{~mm}$ wire mesh on a perforated float made of Styrofoam, and then plunged into liquid nitrogen and stored for at least 1 week. Straws were thawed in a $37^{\circ} \mathrm{C}$ waterbath for $10 \mathrm{~s}$. The thawed semen in each straw was diluted with $1 \mathrm{ml}$ R1ECM supplemented with $0.4 \%(w / v)$ BSA (Fraction V, Sigma) at $37^{\circ} \mathrm{C}$ and then incubated at $37^{\circ} \mathrm{C}$ in $5 \% \mathrm{CO}_{2}$ in humidified air until used for insemination. During all freezing and thawing procedures, spermatozoa were handled gently to avoid physical stress.

\section{Motility and membrane integrity of spermatozoa}

The percentage of motile spermatozoa was assessed visually by direct observation at $37^{\circ} \mathrm{C}$ under a light microscope at $\times 100$. The integrity of the sperm membrane was determined using a commercially available test kit (Live/Dead sperm viability kit, Molecular Probes, Inc., OR) that differentiates between spermatozoa with intact plasma membranes and those with damaged membranes, according to the fluorescent staining pattern observed with a fluorescence microscope (OLYMPUS, BX-50, Tokyo). The nuclei of spermatozoa with intact plasma membranes show green fluorescence, whereas damaged plasma membranes show orange-red fluorescence. At least 300 spermatozoa were counted in duplicate for each sample under the microscope at $\times 200$.

\section{Intrauterine insemination}

Fifty-nine mature Wistar female rats were mated with vasectomized $\mathrm{BN}$ males of proven sterility to induce pseudopregnancy. Female rats that were kept under light conditions for $12 \mathrm{~h}$ (lights on 06:00-18:00 h) and were confirmed to have mated with a vasectomized male rat were used for insemination. Female rats as recipients of inseminations were mated with vasectomized males on day 0 between 16:00 and 22:00 $\mathrm{h}$ to induce pseudopregnancies. On day 0 between 22:00 and 23:00 h, frozen-thawed semen samples were diluted with R1ECM supplemented with $0.4 \%(\mathrm{w} / \mathrm{v}) \mathrm{BSA}$ and then placed in $5 \%$ $\mathrm{CO}_{2}$ in humidified air until used for insemination. The thawed semen sample was inseminated into the oviductal end of each uterine horn of the recipients. A total of 12-13 recipient female rats for each treatment group was inseminated with frozen-thawed semen. As controls, nonfrozen epididymal spermatozoa were inseminated into 13 females with induced pseudopregnancy. The non-frozen semen sample was collected in R1ECM in the same manner as described above and then incubated at $37^{\circ} \mathrm{C}$ in $5 \% \mathrm{CO}_{2}$ in humidified air until used for insemination. The volume of semen inseminated into each uterine horn was about $50 \mu \mathrm{l}$ and contained 1-3 $\times 10^{6}$ spermatozoa. Semen was handled carefully to avoid physical stress. On the morning of day 22, the inseminated females underwent Caesarean section to confirm pregnancy and normality of the fetuses. Foster mothers who had given birth the day before the Caesareans were performed, nursed several offspring derived from cryopreserved spermatozoa. Rats derived from cryopreserved spermatozoa were mated naturally with normal mature rats derived from non-frozen spermatozoa to confirm their fertility. In addition, six female rats with induced pseudopregnancy were inseminated with semen that had been frozen in media with $0.7 \%$ Equex Stem 
without glycerol in the same manner as described above to confirm nursing of females inseminated surgically and growth of their offspring. Females were fed and observed until weaning.

\section{Statistical analysis}

The results are expressed as mean \pm SEM. Data for motility and membrane integrity of spermatozoa, and pregnancy were analysed by one-way ANOVA. When ANOVA revealed a significant effect, values were compared by Tukey's test. Litter sizes were compared by Student's $t$ test.

\section{Results}

The motility of rat spermatozoa that had been centrifuged at $700 \mathrm{~g}$ for $5 \mathrm{~min}$ was lower compared with non-centrifuged spermatozoa (Fig. 1). In addition, after centrifugation three times the percentage of membrane-intact spermatozoa decreased to approximately $0 \%$ (Fig. 2). The addition of $0.7 \%$ Equex Stem to media containing $6 \%$ glycerol resulted in a significant decrease $(P<0.05)$ in the percentage of motile spermatozoa compared with spermatozoa either frozen without or with 3\% glycerol. However, no significant difference was observed between the motility of spermatozoa frozen in media without glycerol or with 3\% glycerol (Table 1). Despite the presence of $0.7 \%$ Equex Stem, the percentage of membrane-intact spermatozoa was significantly higher $(P<0.01)$ in samples frozen without glycerol than in samples frozen with $3 \%$ glycerol (Table 1 ). Although the addition of $0.7 \%$ Equex Stem to the freezing medium with or without $3 \%$ glycerol did not influence the percentage of motile spermatozoa after freezing and thawing, the percentage of membrane-intact spermatozoa frozen in media without glycerol increased $(P<0.05)$ in the presence of $0.7 \%$ Equex Stem (Table 1 ).

Thirteen female rats were inseminated with non-frozen semen in the same manner as the controls. A total of 145 normal live offspring was obtained from all recipient females inseminated with non-frozen spermatozoa. In contrast, the conception rates and the litter sizes of female rats inseminated with frozen-thawed rat spermatozoa were significantly lower $(P<0.01)$ than those of female rats inseminated with non-frozen spermatozoa (Table 2).

An artificial intrauterine insemination test was used to compare: (i) medium containing 0.7\% Equex Stem either with or without 3\% glycerol; and (ii) the presence or absence of $0.7 \%$ Equex Stem in medium without glycerol. Twenty-five females were inseminated in the top of each uterine horn with semen frozen in medium without glycerol, and 13 females were inseminated with semen frozen in medium with $3 \%$ glycerol in the same manner (Table 2). Forty-one normal live offspring were obtained from nine females inseminated with semen frozen in the absence of glycerol, and four normal live offspring were obtained from three females inseminated with semen frozen

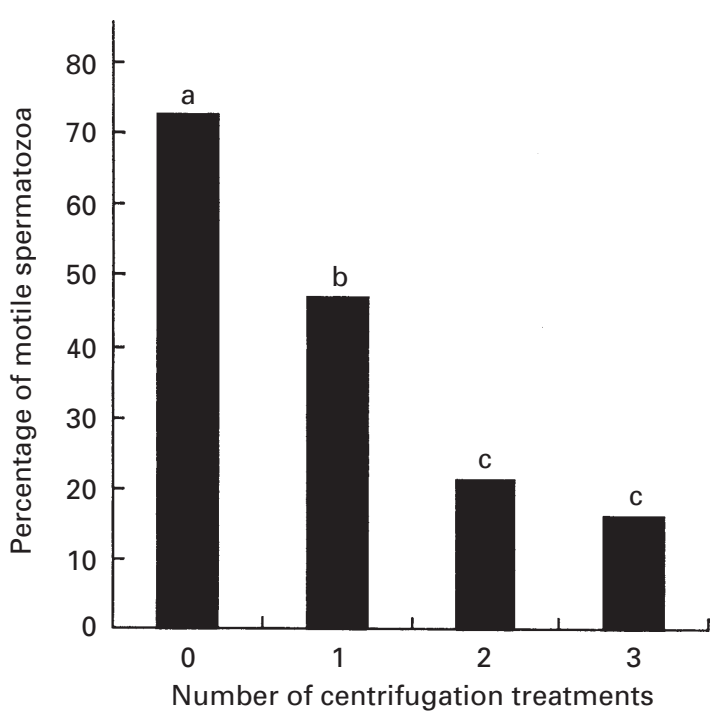

Fig. 1. The effect of the number of centrifugation treatments $(700 \mathrm{~g}$ for $5 \mathrm{~min}$ ) on the motility of rat epididymal spermatozoa. Values with different letters are significantly different $(P<0.05)$.

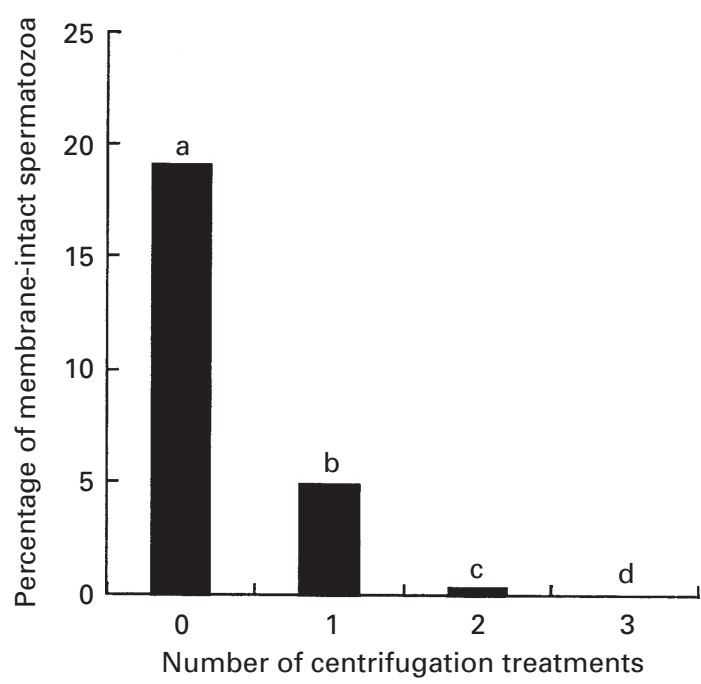

Fig. 2. The effect of the number of centrifugation treatments (700 $\mathbf{g}$ for $5 \mathrm{~min}$ ) on membrane integrity of rat epididymal spermatozoa. Values with different letters are significantly different $(P<0.05)$.

with $3 \%$ glycerol (Table 2). Unexpectedly, the conception rate and litter sizes of females that had been inseminated with spermatozoa frozen in media without glycerol and then thawed were significantly higher $(P<0.05)$ than those of females inseminated with spermatozoa frozen in media containing 3\% glycerol and then thawed. The presence of $0.7 \%$ Equex Stem in the medium did not significantly affect litter sizes. However, the conception rate by artificial intrauterine insemination with frozen-thawed spermatozoa was significantly higher $(P<0.01)$ when using spermatozoa 
Table 1. Effect of glycerol concentrations and the presence of $0.7 \%$ Equex Stem on the motility of frozen-thawed rat epididymal spermatozoa

\begin{tabular}{|c|c|c|c|}
\hline Glycerol (\%) & $\begin{array}{l}\text { Presence of } 0.7 \% \text { Equex } \\
\text { Stem in freezing medium }\end{array}$ & Percentage of motile spermatozoa & $\begin{array}{l}\text { Percentage of membrane-intact } \\
\text { spermatozoa }\end{array}$ \\
\hline \multirow[t]{2}{*}{0} & + & $4.6 \pm 0.5^{\mathrm{a}}$ & $9.2 \pm 3.9^{a}$ \\
\hline & - & $4.7 \pm 0.2^{\mathrm{a}}$ & $5.1 \pm 4.0^{\mathrm{bd}}$ \\
\hline \multirow[t]{2}{*}{3} & + & $4.3 \pm 0.6^{\mathrm{a}}$ & $2.8 \pm 0.9^{c}$ \\
\hline & - & $3.4 \pm 0.5^{\mathrm{ab}}$ & $1.3 \pm 1.1^{\mathrm{ce}}$ \\
\hline 6 & + & $2.9 \pm 0.4^{b}$ & $0.8 \pm 1.4^{\text {ce }}$ \\
\hline
\end{tabular}

Values are means \pm SEM.

Values within a column with different superscripts are significantly different $(P<0.05)$.

Semen samples from 41 donor male rats were assigned randomly to each group (7-8 cryopreserved samples per group).

Table 2. Intrauterine insemination of female rats with epididymal spermatozoa cryopreserved either with or without glycerol and with or without $0.7 \%$ Equex Stem

\begin{tabular}{|c|c|c|c|c|c|c|c|}
\hline \multirow[b]{2}{*}{$\begin{array}{l}\text { Inseminated } \\
\text { spermatozoa }\end{array}$} & \multicolumn{2}{|c|}{ Cryoprotective agents } & \multirow[b]{2}{*}{$\begin{array}{l}\text { Sperm motility } \\
(\%)^{*}\end{array}$} & \multirow[b]{2}{*}{$\begin{array}{c}\text { Pregnancy } \\
(\%)\end{array}$} & \multirow{2}{*}{$\begin{array}{c}\text { Total number of } \\
\text { fetuses on day } 22 \\
\text { of pregnancy }\end{array}$} & \multicolumn{2}{|c|}{ Litter size } \\
\hline & Glycerol (\%) & $\begin{array}{c}0.7 \% \text { Equex } \\
\text { Stem }\end{array}$ & & & & $\begin{array}{l}\text { Pregnant } \\
\text { females }\end{array}$ & $\begin{array}{c}\text { All inseminated } \\
\text { females }\end{array}$ \\
\hline Non-frozen & & & $75.0 \pm 7.7^{a}$ & $13 / 13(100)^{a}$ & 145 & $11.2 \pm 2.2^{\mathrm{a}}$ & $11.2 \pm 2.2^{\mathrm{a}}$ \\
\hline Frozen-thawed & $\begin{array}{l}0 \\
0 \\
3\end{array}$ & $\begin{array}{l}+ \\
- \\
+\end{array}$ & $\begin{array}{l}9.0 \pm 3.3^{b} \\
9.6 \pm 1.5^{b} \\
7.0 \pm 1.2^{b}\end{array}$ & $\begin{array}{l}9 / 13(69.2)^{b} \\
5 / 12(41.7)^{c} \\
3 / 13(23.1)^{d}\end{array}$ & $\begin{array}{r}41 \\
13 \\
4\end{array}$ & $\begin{array}{l}4.6 \pm 4.9^{b c} \\
2.6 \pm 1.4^{b} \\
1.0 \pm 0^{b d}\end{array}$ & $\begin{array}{l}3.2 \pm 4.6^{b c} \\
1.1 \pm 2.3^{b} \\
0.2 \pm 4.3^{b d}\end{array}$ \\
\hline
\end{tabular}

*Sperm motility was assessed at insemination.

Semen samples from 47 donor male rats were assigned randomly to each treatment group (7-8 cryopreserved samples per group).

Values within a column with different superscripts differ significantly $(P<0.05)$.

frozen in medium with $0.7 \%$ Equex Stem than in medium without Equex Stem (Table 2).

The fertility of each rat born as a result of frozen-thawed spermatozoa insemination was confirmed as follows: foster mothers nursed eight females and two males produced after artificial insemination of female rats with cryopreserved and thawed spermatozoa, and their fertility was confirmed by a progeny test when they matured (data not shown). Female rats that had been surgically inseminated with frozenthawed spermatozoa were able to deliver their young naturally. Four of six females that had been inseminated with cryopreserved and thawed spermatozoa delivered a total of 17 live young naturally (litter sizes: 8, 5, 3 and 1) and three of these female rats nursed 16 young until weaning, with the exception of the female that produced only one pup which died.

\section{Discussion}

Rat epididymal spermatozoa are extremely sensitive to physical stress, such as centrifugation and other in vitro procedures. Therefore, a key to the successful development of the cryopreservation procedure was to minimize stress at each step.
In the present study, the basic medium used for cryopreservation of rat spermatozoa was the medium used by Kikuchi et al. (1999) for cryopreservation of boar semen. This medium was chosen because (i) boar spermatozoa are particularly sensitive to cooling and freezing; and (ii) the egg yolk in the freezing medium is widely used for sperm preservation because of its beneficial effect on fertility in semen extenders for domestic animals (Benson et al., 1967). In addition, this medium exerts protective properties against cold shock and freezing to preserve motility and membrane integrity of spermatozoa, and it also acts as an osmotic buffer (Watson, 1995). As cryoprotective agents for freezing rat spermatozoa, various concentrations of glycerol either in the presence or absence of $0.7 \%$ Equex Stem were added to the freezing medium to determine the optimum conditions for cryopreservation. Glycerol has cryoprotective properties as reported by Polge et al. (1949) and has been used extensively as a cryoprotective agent for various cells, including mammalian spermatozoa and embryos. In the present study, glycerol, rather than acting as a cryoprotectant, exerted a detrimental effect on motility, membrane integrity and fertility of frozen-thawed spermatozoa inseminated into female rats. As the cryoprotective agents could not be eliminated from the medium by centrifugation 
after thawing, sperm motility was assessed immediately after thawing in the presence of the cryoprotective agents. Equex Stem, which is a surfactant, is a beneficial component of the medium for freezing boar semen (Pursel et al., 1978). The cryoprotective effect of Equex Stem on the rates of membrane integrity and fertility of frozen-thawed rat spermatozoa was confirmed in the present study. Thus, the results of the present study indicate that a medium without glycerol but with $0.7 \%$ Equex Stem is suitable for cryopreservation of rat spermatozoa.

There was a significant difference between the fertility of fresh and frozen-thawed rat spermatozoa when used for surgical insemination. Therefore, it is necessary to improve the technique for freezing and thawing of rat spermatozoa. Nevertheless, the cryopreservation method developed in the present study may be satisfactory. Further investigation is needed to improve the cryopreservation procedures.

It is unclear why cryopreservation of rat spermatozoa is more difficult than that of other mammals. In mice, the birth of live young derived from frozen-thawed semen has been reported (Tada et al., 1990; Yokoyama et al., 1990; Nakagata and Takeshima, 1992; Songsasen et al., 1997). The apparent extreme sensitivity of mouse spermatozoa to freezing has been attributed to a low water permeability coefficient and a long sperm tail as compared with spermatozoa of domestic animals (Noiles et al., 1995). As rat spermatozoa have longer tails than mouse spermatozoa (Clouthier et al., 1996), it is possible that rat spermatozoa are more susceptible to freezing injury than mouse spermatozoa.

In conclusion, the present study demonstrates that live young can be produced from rat spermatozoa frozen at $-196^{\circ} \mathrm{C}$ when used for surgical insemination. In the present study, centrifugation was not used during collection of spermatozoa or during freezing and thawing of spermatozoa, and the medium used to cryopreserve spermatozoa contained egg yolk, lactose and Equex Stem. It was possible to use this method to carry out freezing, thawing and artificial intrauterine insemination of rat spermatozoa to litters that were shown to be capable of producing young. The sperm cryopreservation procedure described in this study offers a reliable method for cryopreservation of rat spermatozoa in liquid nitrogen. This novel method should be efficient for preserving genetic sources of mutant or transgenic rats. To our knowledge, the method developed in the present study is the first successful production of offspring using rat spermatozoa cryopreserved in liquid nitrogen.

This research was supported in part by Grants-in-Aid Scientific Research (No. 11356008 and No. 12680817) from the Ministry of
Education, Science, Sports and Culture of Japan and by a grant from Science Research Promotion Fund from the Promotion and Mutual Aid Corporation for Private Schools of Japan.

\section{References}

An TZ, Iwakiri M, Edashige K, Sakurai T and Kasai M (2000) Factors affecting the survival of frozen-thawed mouse spermatozoa Cryobiology 40 237-249

Benson RW, Pickett BW, Komarekm RJ and Lucas JJ (1967) Effect of incubation and cold shock on motility of boar spermatozoa and their relationship to lipid content Journal of Animal Science 26 1078-1081

Clouthier DE, Avarbock MR, Maika SD, Hammer RE and Brinster RL (1996) Rat spermatogenesis in mouse testis Nature 381 418-421

Kikuchi K, Kashiwazaki N, Nagai T et al. (1999) Reproduction in pigs using frozen-thawed spermatozoa from epididymis stored at $4{ }^{\circ} \mathrm{C}$ Journal of Reproduction and Development 45 345-350

Miyoshi K, Kono T and Niwa K (1997) Stage-dependent development of rat 1 -cell embryos in a chemically defined medium after fertilization in vivo and in vitro. Biology of Reproduction 56 180-185

Nakagata N (1996) Use of cryopreservation techniques of embryos and spermatozoa for production of transgenic ( $\mathrm{Tg}$ ) mice and for maintenance of Tg mouse lines Laboratory Animal Science 46 236-238

Nakagata $\mathbf{N}$ and Takeshima $\mathbf{T}$ (1992) High fertilizing ability of mouse spermatozoa diluted slowly after cryopreservation Theriogenology 37 1283-1291

Nakagata N, Okamoto M, Ueda O and Suzuki H (1997) Positive effect of partial zona-pellucida dissection on the in vitro fertilizing capacity of cryopreserved $\mathrm{C} 57 \mathrm{BL} / 6$ J transgenic mouse spermatozoa of low motility Biology of Reproduction 57 1050-1055

Noiles EE, Bailey JL and Storey BT (1995) The temperature dependence in the hydraulic conductivity, $\mathrm{L}_{\mathrm{pP}}$, of the mouse plasma membrane shows a discontinuity between 4 and $0^{\circ} \mathrm{C}$ Cryobiology 32 220-238

Polge C, Smith AU and Parkes AS (1949) Revival of spermatozoa after vitrification and dehydration at low temperatures Nature 164 666-668

Pursel VG, Schulman LL and Johnson LA (1978) Effect of Orvus ES Paste on acrosome morphology, motility and fertilizing capacity of frozen-thawed boar sperm Journal of Animal Science 47 198-202

Songsasen N, Betteridge KJ and Leibo SP (1997) Birth of live mice resulting from oocytes fertilized in vitro with cryopreserved spermatozoa Biology of Reproduction $\mathbf{5 6}$ 143-152

Sztein JM, Farley JS and Mobraaten E (2000) In vitro fertilization with cryopreserved inbred mouse sperm Biology of Reproduction 63 1774-1780

Tada N, Sato M, Yamanoi J, Mizorogi T, Kasai K and Ogawa S (1990) Cryopreservation of mouse spermatozoa in the presence of raffinose and glycerol Journal of Reproduction and Fertility 89 511-516

Watson PF (1995) Recent developments and concepts in the cryopreservation of spermatozoa and the assessment of their post thawing function Reproduction, Fertility and Development 7 871-891

Yokoyama M, Akiba H, Katsuki M and Nomura T (1990) Production of normal young following transfer of mouse embryos obtained by in vitro fertilization using cryopreserved spermatozoa Experimental Animals 39 125-128

Received 5 February 2001.

First decision 9 March 2001.

Accepted 2 May 2001. 